\title{
Conditionally exactly soluble class of quantum potentials
}

\author{
Alvaro de Souza Dutra \\ UNESP-Campus de Guaratinguetá-DFQ, Avenida Dr. Ariberto Pereira da Cunha 333, CEP 12500 Guaratinguetá, São Paulo, Brazil* \\ and Centro Brasileiro de Pesquisas Físicas-DCP, Rua Dr. Xavier Sigaud 150, CEP 22290-180 Urca, Rio de Janeiro, Brazil
}

(Received 23 June 1992)

\begin{abstract}
We present a different class of quantum-mechanical potentials. These are midway between the exactly solvable potentials and the quasiexactly ones. Their fundamental feature is that one can find the entire $s$-wave spectrum of a given potential, provided that some of its parameters are conveniently fixed.

PACS number(s): 03.65.Ca, 03.65.Ge, 02.90. $+\mathrm{p}$
\end{abstract}

Since the appearance of quantum mechanics, there has been continual searching for exactly soluble (ES) potentials [1]. This relies on the importance of such solutions in many branches of physics, and on the fact that these solutions can be used as a basis to perform perturbative calculations in nonexact potentials. Until recently it was thought that there would exist only two classes of potentials in quantum mechanics, namely, the exactly soluble and the nonexactly soluble ones. However, about a decade ago [2], another class was discovered and, since then, has been widely discussed in its various aspects [3-7]. This class, the so-called quasiexactly soluble (QES) potentials, is characterized by the fact that it is only possible to have an exact finite number of their energy eigenstates: the remaining eigenstates can only be obtained through numerical calculations, as with the nonexactly soluble potentials. The importance in the study of these potentials, apart from intrinsic academic interest, rests on the possibility of using their solutions to test the quality of numerical methods and in the possible existence of real physical systems that they could represent.

Here we intend to report the finding of another class of potentials, what we call the conditionally exactly soluble (CES) potentials, because its principal feature is that of having exact solutions only when certain conditions are made, namely, that some of the parameters of the potential be fixed to a very specific value.

This class of potentials is positioned between the ES and the QES potentials. All of their energy levels can be exactly obtained, as with the ES potentials, but their parameters cannot be arbitrarily chosen, as with the QES potentials. The first feature put them in conditions to be used in perturbative calculations. having the advantage that, for the potentials belonging approximately to their form, they take into account the anharmonicity of the potential, unlike the traditional perturbative method where the wave functions of the harmonic oscillator or any other exact potential with a more appropriate form are commonly used. The second feature put the CES potentials in a more suitable situation, when compared with the $Q$ potentials, in order to testify about the quality of a given numerical approach. Furthermore, we will see that one could expect some fashion of reality in at least one of these potentials.

The way to obtain such potentials as we use is to look for a mapping between them and a driven harmonic oscillator. This is done by performing nonlinear coordinate transformations, and then requiring that a certain term vanish. We start with the Schrödinger equation for a given potential $V(r)$, perform the variable transformation $r=f(u)$, and redefine the wave function as

$$
\psi(r, t)=\sqrt{f^{\prime}(u(r))} \chi(r, t),
$$

where the prime denotes differentiation with respect to the variable $u$. This new wave function obeys the transformed Schrödinger equation

$$
\left\{-\frac{\bar{h}^{2}}{2 \mu} \frac{\partial^{2}}{\partial u^{2}}+V_{T}(u)\right\} \chi(u)=E_{T} \chi(u),
$$

where $\mu$ is the mass, and $E_{T}$ is some constant resulting from the transformation that takes the role of the "energy" in the new equation. Furthermore, it is straightforward to show that

$$
V_{T}(u)-E_{T}=\left(f^{\prime}(u)\right)^{2}[V(f(u))-E]+\Delta V(u),
$$

with

$$
\Delta V(u)=\frac{\hbar^{2}}{\mu}\left[-\frac{1}{4} \frac{f^{\prime \prime \prime}(u)}{f^{\prime}(u)}+\frac{3}{8}\left[\frac{f^{\prime \prime}(u)}{f^{\prime}(u)}\right]^{2}\right] .
$$

This type of approach has been used extensively in the path-integral method of quantization [8]. In fact, the above procedure was also used in the Schrödinger picture to relate different power-law potentials [9], and also to relate spherically symmetric potentials at different space dimensions [10]. However, for transformations where $f(u)=u^{\alpha}$, with $\alpha$ being real, $\Delta V(u)$ will always produce a $u^{-2}$ term, which should be removed in order to get a driven harmonic oscillator potential in the new variable. This is the origin, for the cases here considered, of the very particular fixing of one of the potential parameters. At this point it is interesting to make some considerations about the boundary conditions after the transformation $r=f(u)$. The principal care to be taken is with respect to the normalizability of the wave function, in order to maintain the normalization of the final wave function after returning to the physical variable.

Let us now look at the first two representatives of this new class of potentials. First of all we see that an exten- 
sion of our original Coulomb potential belongs to it. This potential is

$$
V(r)=\frac{A}{r}+\frac{B}{r^{1 / 2}}+\frac{G_{0}}{r^{2}} .
$$

The transformation is the same one that links the Coulomb potential with a three-dimensional harmonic oscillator $f(u)=u^{2}$ [8]. In this case the expression (2) reads

$$
V_{T}(u)-E_{T}=-4 E u^{2}+4 B u+4 A+\left[4 G_{0}+\frac{3 \hbar^{2}}{8 \mu}\right] u^{-2},
$$

where we can identify $E_{T}$ with $4 A$, the frequency of the oscillator $\omega=\sqrt{-8 E / \mu}$, and the driving force is $4 B$. In order to get an exact solution one can follow one of two possible ways. The first one is the elimination of the driving force $(B=0)$, leading to the solution of the usual Coulomb potential. The second way is that of imposing the elimination of the centrifugal-barrier-type term; this is obtained by imposing that $G_{0}=-3 \hbar^{2} / 32 \mu$. This last case led us to the first CES potential presented in this work. It is interesting to observe that for great distances it behaves like an $s$-wave Coulomb potential.

However, before obtaining the solution of the problem, let us make a further transformation to map the CES potential into the harmonic oscillator. Using the translation $v=u-B /(2 E)$, we get

$$
V_{T}(v)-E_{T}=-4 E v^{2}+4 A+B^{2} / E .
$$

Now using the solution for the spectrum of the harmonic oscillator, we get the following equation for the spectrum of the CES potential:

$$
E_{T}=(n+1 / 2) \hbar \omega
$$

so that

$$
4 A+B^{2} / E_{n}=(n+1 / 2) \hbar \sqrt{-8 E_{n} / \mu},
$$

which after simple manipulations leads us to the equation for $E_{n}$

$\hbar^{2}(n+1 / 2)^{2} E_{n}^{3}+2 \mu A^{2} E_{n}^{2}+\mu A B^{2} E_{n}+\mu B^{4} / 8=0$.

On the other hand, using the solutions of the eigenstates of the harmonic oscillator, returning to the variable $r$, and using (1), we obtain the eigenfunctions

$$
\begin{aligned}
\psi_{n}(r)= & \left(2^{(n-1)} n !\right)^{-1 / 2}\left(\frac{r \beta_{n}^{2}}{\pi}\right)^{1 / 4} H_{n}\left(\beta_{n}\left(r^{1 / 2}-\frac{B}{2 E_{n}}\right)\right] \\
& \times \exp \left[-\frac{\beta_{n}^{2}}{2}\left[r^{1 / 2}-\frac{B}{2 E_{n}}\right]^{2}\right]
\end{aligned}
$$

where we defined

$$
\beta_{n} \equiv\left(-\frac{8 \mu E_{n}}{\hbar^{2}}\right)^{1 / 4}
$$

From the three solutions of the spectrum equation (8) we shall discard two. This can be done by a simple physical criterion. As can be seen from the form of the potential, we see that, when $B=0$, the energy spectrum must be reduced to one of the Coulomb type. Using this criterion one gets the solution

$E_{n}=\left[R+\left(Q^{3}+R^{2}\right)^{1 / 2}\right]^{1 / 3}+\left[R-\left(Q^{3}+R^{2}\right)^{1 / 2}\right]^{1 / 3}-\frac{a_{1}}{3}$,

where

$$
Q \equiv\left(3 a_{2}-a_{1}^{2}\right) / 9, \quad R \equiv\left(9 a_{1} a_{2}-27 a_{3}-2 a_{1}^{3}\right) / 54,
$$

and

$$
\begin{aligned}
& a_{1}=\frac{2 \mu A^{2}}{\hbar^{2}(n+1 / 2)^{2}}, a_{2}=\frac{\mu A B^{2}}{\hbar^{2}(n+1 / 2)^{2}}, \\
& a_{3}=\frac{\mu B^{4}}{8 \hbar^{2}(n+1 / 2)^{2}} .
\end{aligned}
$$

The second example of a CES potential, as far as we know, does not have in some limit any other well-known ES potential, as happened in the previous example. The potential appears like

$$
V(r)=A r^{2 / 3}+\frac{B}{r^{2 / 3}}+\frac{g_{0}}{r^{2}},
$$

where $g_{0}$ is chosen to be equal to $-5 \hbar^{2} / 72 \mu$, in a completely analogous fashion to the previous case. It is remarkable to observe that a nonexact version of this potential was considered recently [11], in connection with an effective quark-antiquark potential model for heavy and light mesons. In fact, it is easy to see that for high radial quantum numbers the above CES potential will have its spectrum be closer and closer to the nonexact case appearing in [11]. The transformation function in this case is $f(u)=u^{3 / 2}$, Eq. (3) looks like

$$
V_{T}(u)-E_{T}=(9 A / 4) u^{2}-(9 E / 4) u+9 B / 4,
$$

and now we identify $E_{T}$ with $9 B / 4, \omega=\sqrt{9 A / 2 \mu}$, and the driving force is represented by $-9 E / 4$. Using a procedure analogous to the previous case we see that this time the eigenfunctions are

$$
\begin{aligned}
\psi_{n}(r)= & \left(2^{(n+1)} n ! / 3\right)^{-1 / 2}\left[\frac{r^{2 / 3} \beta^{2}}{\pi}\right]^{1 / 4} \\
& \times H_{n}\left[\beta\left[r^{2 / 3}-\frac{E_{n}}{2 A}\right]\right) \\
& \times \exp \left[-\frac{\beta^{2}}{2}\left[r^{2 / 3}-\frac{E_{n}}{2 A}\right]^{2}\right],
\end{aligned}
$$

with $\beta \equiv\left(9 \mu A / 2 \hbar^{2}\right)^{1 / 4}$. The equation for the spectrum is simply

$$
E_{n}^{2}=\frac{16 A}{9}[(n+1 / 2) \sqrt{9 A / 2 \mu}+9 B / 4],
$$

with the solutions 
$E_{n}= \pm \frac{4 \sqrt{A}}{3}[(n+1 / 2) \sqrt{9 A / 2 \mu}+9 B / 4]^{1 / 2}$

It is not difficult to convince oneself that, for the cases where the parameter $B$ is positive, the positive solution is quite good, but if $B$ is negative one can see that there will exist prohibited quantum numbers. This can be seen by imposing that $E_{n}$ must be a real quantity, so

$$
n \geq \operatorname{Int}\left[\left(\frac{9 B^{2}}{8 A}\right]^{1 / 2}-\frac{1}{2}\right],
$$

where Int [ ] stands for the first integer after the value of its argument. This, however, does not say that there are not such energy levels, but that perhaps they should be searched for in the negative solutions in (14b). It is only a matter of experimentation to verify that, for instance, with $n=1$ the negative solution looks like a ground-state eigenfunction, because it does not have any node.

The problem is that this potential, in contrast with the former, does not have some type of limit as a guide for deciding about what solution to use. This problem is under investigation and we intend to report on it in a further publication.

As should be expected, these two cases can be mapped one onto another through a suitable transformation: in the first one, for example, it would be $f(u)=u^{4 / 3}$. Some extensions can be thought of as looking for supersymmetric partners; one can find other CES representative potentials.

We can also study the possibility of applying the second potential presented above to the case of quarkantiquark potential models. These and other problems related to the CES potential are presently under investigation, and we expect to report on them in the near future.

The author thanks the CNPq (Conselho Nacional de Desenvolvimento Científico e Technológico) of Brazil for partial financial support.
*Permanent address.

[1] S. Flügge, Practical Quantum Mechanics (Springer, Berlin, 1971).

[2] G. P. Flessas, Phys. Lett. A 72, 289 (1979); 78, 19 (1980); 81, 17 (1981); J. Phys. A 14, L209 (1981).

[3] P. G. L. Leach, J. Math. Phys. 25, 974 (1984); Physica D 17, 331 (1985).

[4] A. de Souza Dutra, Phys. Lett. A 131, 319 (1988).

[5] A. V. Turbiner, Commun. Math. Phys. 118, 467 (1988).
[6] M. A. Shiffman, Int. J. Mod. Phys. A 4, 2897 (1989).

[7] A. de Souza Dutra and H. Boschi Filho, Phys. Rev. A 44, 4721 (1991), and references therein.

[8] N. K. Pak and I. Sökmen, Phys. Rev. A 30, 1629 (1984); J. M. Cai and A. Inomata, Phys. Lett. A 141, 315 (1989), and references therein.

[9] R. Johnson, J. Math. Phys. 21, 2640 (1980).

[10] E. Papp, Phys. Rev. A 38, 5910 (1988).

[11] X. Song, J. Phys. G 17, 49 (1991). 